\section{Outbreak of Burkholderia cepacia Bacteremia Caused by Contaminated Chlorhexidine in a Hemodialysis Unit}

TO THE EDITOR-Burkholderia cepacia is a widespread gram-negative environmental bacillus associated with nosocomial infection. ${ }^{1,2}$ This organism colonizes water supplies, filter membranes, and antiseptic solutions. ${ }^{3-5}$ Inadequate catheter care, defects in membrane integrity, and the reprocessing of dialyzers have all been implicated in outbreaks in hemodialysis units. ${ }^{6,7}$ We describe an outbreak of $B$. cepacia bacteremia that occurred in Madrid, Spain, in the Alcobendas hemodialysis center.

The Alcobendas hemodialysis center has 14 stations and serves approximately 60 outpatients with chronic renal failure per month. Municipal water passes through cartridge filters, water softeners, carbon filters, and a simple reverse-osmosis membrane unit before being distributed to the dialysis stations in a closed-loop circuit. Dialysis machines are disinfected according to the manufacturer's instructions at the end of each dialysis session.

Chlorhexidine was purchased by the hemodialysis center as a $5 \%$ solution and diluted with deionized water to a $2.5 \%$ solution, then stored in $250-\mathrm{mL}$ plastic bottles. The solution was prepared every 7-10 days by healthcare workers. The $2.5 \%$ chlorhexidine solution was used to disinfect the skin of patients before catheter insertion and during follow-up care.

The nosocomial outbreak of $B$. cepacia bacteremia occurred from December 1, 2005, through April 30, 2006. Five outpatients developed symptoms of bacteremia. The overall attack rate was $1.6 \%$. An outbreak case was defined by the presence of fever and the detection of $B$. cepacia in blood cultures.

Four of the case patients had long-term central venous catheters (CVCs) and developed high temperatures without signs of catheter-related infection. The catheters were removed from all 4 patients but only 1 catheter was sent to the laboratory for culture.

The unusually high number of $B$. cepacia isolates motivated us to carry out an epidemiological study. Blood samples are usually inoculated into both aerobic and anaerobic media for processing with the Versatrek culture system (Trek Diagnostic Systems). The blood samples were cultured on 3 agar plates: sheep blood agar, chocolate blood agar, and brucella agar. The sheep blood agar and chocolate blood agar plates were incubated at $35^{\circ} \mathrm{C}$ in an atmosphere containing $5 \% \mathrm{CO}_{2}$ for 48 hours. The brucella agar was incubated at $35^{\circ} \mathrm{C}$ in an anaerobic atmosphere for 48 hours.

Environmental cultures were performed on potential sources of $B$. cepacia contamination: the deionized water used to dilute the dialysate concentrate, the postosmosis water, the tap water of the 14 hemodialysis stations, the undiluted $5 \%$ chlorhexidine solution, the diluted $2.5 \%$ chlorhexidine solution, and the povidone-iodine solution. All environmental samples were cultivated on $B$. cepacia-selective agar and incubated at $35^{\circ} \mathrm{C}$ in an atmosphere containing $5 \% \mathrm{CO}_{2}$ for 18 hours.

Biochemical identification was performed with the Vitek 2 GNI card (bioMérieux Vitek) and Biolog GN2 panels (Biolog). Susceptibility testing was performed with the Wider System (Francisco Soria Melguizo). Genotyping of clinical and environmental $B$. cepacia strains was performed after digestion with the restriction enzyme $\mathrm{XbaI}$ with the $\mathrm{CHEF}$ DR-III system (BioRad) according to the conditions previously described. ${ }^{8}$

During the outbreak, B. cepacia was isolated from the blood of 5 patients treated at the hemodialysis unit and from 2 environmental samples. This organism was detected in the $2.5 \%$ chlorhexidine solution and in the water used to dilute the chlorhexidine at station 3. Other potential sources of contamination yielded no $B$. cepacia.

All the clinical and environmental isolates were of Burkholderia stabilis sp. nov. (formerly Burkholderia cepacia genomovar IV) and had identical DNA banding patterns, as determined by pulsed-field gel electrophoresis (PFGE), suggesting their relation and a common source of infection. The clonality of the 7 isolates is shown in the Figure.

Our findings suggest that the outbreak of $B$. cepacia bacteremia in patients receiving long-term hemodialysis at our institution was caused by the use of contaminated alcoholfree $2.5 \%$ chlorhexidine solution for skin disinfection before catheter insertion and during follow-up care. All the patients of the hemodialysis unit were exposed to this solution, but only 5 women, with a median age of 79 years (range, 77-81 years), developed infection. Concomitant long-term use of a CVC and advanced age were significant risk factors.

We hypothesize that the water tap of station 3 was contaminated with the $B$. cepacia through inappropriate manipulation when the $2.5 \%$ chlorhexidine solution was prepared by healthcare workers, because samples of water from the rest of the distribution system in the hemodialysis unit yielded no B. cepacia. After we discarded the diluted chlorhexidine solution and cleaned the tap of station 3 with solutions of sodium hypochlorite and $80 \% \mathrm{v} / \mathrm{v}$ ethanol, no further cases were identified.

Environmental bacteria are becoming increasingly involved in outbreaks. In these cases, molecular identification and typing are fundamental for determining the correct strategy in the management of epidemics.

On the basis of evidence from our study and others in the literature, we recommend that hospitals and other hemodi- 


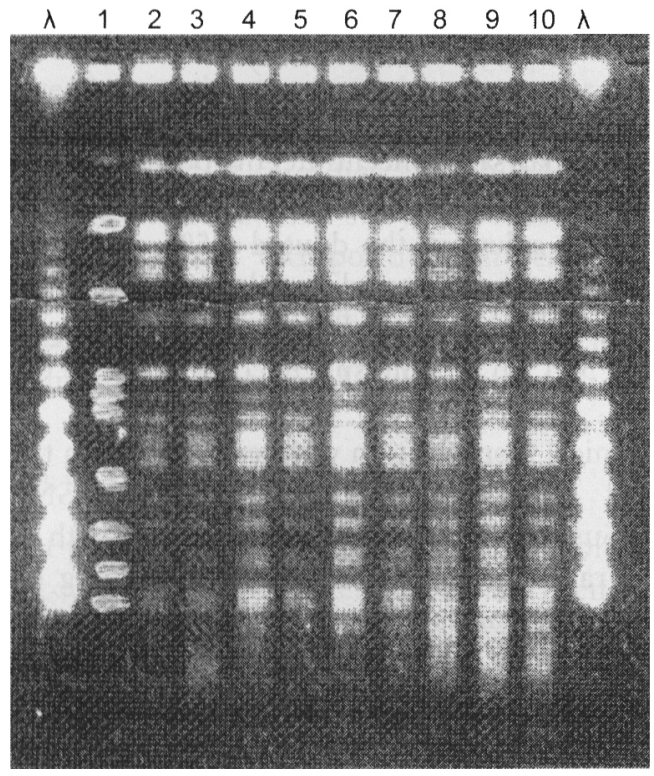

FIGURE. Pulsed-field gel electrophoresis (PFGE) of $B$. cepacia strains. The $\lambda$ denotes the lambda ladder PFGE weight marker (48.5 kbp concatemers). Lane 1 , an epidemiologically unrelated strain recovered from a cystic fibrosis patient; Lane 2, strain retrieved from the catheter of patient 2; Lanes 3-7, strains isolated from the blood of patients 1, 2, 3, 4, and 5, respectively; Lane 8, strain recovered from a sample of the tap water of hemodialysis station 3; Lanes 9 and 10 , strains recovered from 2 bottles of $2.5 \%$ chlorhexidine solution.

alysis units discontinue the use of diluted alcohol-free chlorhexidine solution. In fact, the manufacturer's instructions recommend adding alcohol when diluting the chlorhexidine for skin disinfection before catheter insertion and during follow-up care.

\section{ACKNOWLEDGMENTS}

Potential conflicts of interest. All authors report no conflicts of interest relevant to this study.

M. P. Romero-Gómez, MD; M. I. Quiles-Melero, MD; P. Peña García, MD; A. Gutiérrez Altes, MD; M. A. García de Miguel, MD; C. Jiménez, MD; Sylvia Valdezate, $\mathrm{PhD}$; J. A. Sáez Nieto, $\mathrm{PhD}$

From the Departments of Microbiology and Parasitology (M.P.R.-G., M.I.Q.-M., P.P.G., A.G.A.) and Nephrology (M.A.G.M., C.J.), La Paz University Hospital, and the Servicio de Bacteriología, Centro Nacional de Microbiología, Instituto de Salud Carlos III, Majadahonda (S.V., J.A.S.N.), Madrid, Spain.

Address reprint requests to M. P. Romero-Gomez, Department of Microbiology and Parasitology, La Paz University Hospital, Paseo de la Castellana, 261, 28046 Madrid, Spain (maryfirm2000@msn.com).

Infect Control Hosp Epidemiol 2008; 29:377-378

(C) 2008 by The Society for Healthcare Epidemiology of America. All rights reserved. 0899-823X/2008/2904-0018\$15.00. DOI: $10.1086 / 529032$

\section{REFERENCES}

1. Humar A, Oxley C, Sample ML, Garner G. Elimination of an outbreak of gram-negative bacteremia in a hemodialysis unit. Am J Infect Control $1996 ; 24: 359-363$.

2. Lu DC, Chang SC, Chen YC, Luh KT, Lee CY, Hsieh WC. Burkholderia cepacia bacteremia: a retrospective analysis of 70 episodes. J Formos $\mathrm{Med}$ Assoc 1997; 96:972-978.

3. Reboli AC, Koshinski R, Arias K, Marks-Austin K, Stieritz D, Stull TL. An outbreak of Burkholderia cepacia lower respiratory tract infection associated with contaminated albuterol nebulization solution. Infect Control Hosp Epidemiol 1996; 17:741-743.

4. Molina-Cabrillana J, Bolaños-Rivero M, Alvarez-León EE, et al. Intrinsically contaminated alcohol-free mouthwash implicated in a nosocomial outbreak of Burkholderia cepacia colonization and infection. Infect Control Hosp Epidemiol 2006; 27:1281-1282.

5. Ghazal SS, Al-Mudaimeegh K, Al Fakihi EM, Asery AT. Outbreak of Burkholderia cepacia bacteremia in immunocompetent children caused by contaminated nebulized sulbutamol in Saudi Arabia. Am I Infect Control 2006; 34:394-398.

6. Kaitwatcharachai C, Silpapojakul K, Jitsurong S, Kalnauwakul S. An outbreak of Burkholderia cepacia in hemodialysis patients: an epidemiologic and molecular study. Am I Kidney Dis 2000; 36:199-204.

7. Magalhaes M, Doherty C, Govan JR, Vandamme P. Polyclonal outbreak of Burkholderia cepacia complex bacteraemia in haemodialysis patients. $J$ Hosp Infect 2003; 54:120-123.

8. Anderson DJ, Kuhns IS, Vasil ML, Gerding DN, Janoff EN. DNA fingerprinting by pulsed field gel electrophoresis and ribotyping to distinguish Pseudomonas cepacia isolates from a nosocomial outbreak. J Clin Microbiol $1991 ; 29: 648-649$.

\section{Outbreak of Pseudomonas aeruginosa Infections Associated With Contaminated Water in a University Hospital in Tunisia}

To the Editor-Pseudomonas aeruginosa is a major pathogen that causes nosocomial infections, particularly in ventilated and/or immunocompromised patients. This organism is ubiquitous in moist environments and is frequently found in various hospital sites. Strains involved may be spread via the hands of healthcare workers or by an environmental source, such as contaminated water. ${ }^{1-5}$ The installation of surveillance systems that detect outbreaks of nosocomial infection is important; sources of infection may be characterized and isolated, and modifications in procedures made to stop further infections from occurring. Infection is a frequent event in surgical wards, such as the urology ward, where endoscopy and surgical procedures are common.

We describe an outbreak of $P$. aeruginosa infection that occurred between July and September 2005 in the urology ward in the University Hospital Sahloul in Tunisia, a 548bed hospital. An investigation of the environment was done to determine the potential source of infection and to implement control measures to stop the outbreak. The urology 